\title{
Research on Simulation and Calculation of a New Reconfigurable Sea Water Antenna
}

\author{
Xi Chen \\ China Ship Development and Design Center, Wuhan 430064, China. \\ chx_sm@126.com
}

\begin{abstract}
In this paper, a new reconfigurable antenna, a sea water antenna, is constructed and simulated to analyze the its radiation performance by applying Soft CST. Firstly, after analyzing its material structure and properties, a simulated model for the sea antenna is established. Secondly, the effects of the length of the sea water antenna, its diameter, its feeding configuration and sea water sality and conduction on reflected wave, frequency drift and radiation efficiency are analyzed and simulated. Finally, after analyzed these results three practicable sea antennas and its parameters are presented. The proposed antenna and configuration parameters have great benefit to the design and application in practice, especially in the ship antenna field.
\end{abstract}

Keywords: antenna simulation, Configurable antenna, Sea water antenna, Antenna technology.

\section{Introduction}

There are hundreds of kinds of antennas needed in modern naval vessel (ship) in order for communicating, however the space in ship is limited, which makes it difficult to find enough space to place antennas on the premise of having no interference with each other. Even if there is enough space in ships, a great number of antennas, some of which have a large geometry size, has a great influence on RCS of the ship to make it easy to be detected by enemy's radar. So many developed countries in the world are developing new antennas to save space and be stealthy, without reducing the performance of antennas. In the nowadays, some reconfigurable antennas and integrated miniature antennas such as enclosed mast antennas, liquid antennas or seawater antennas are invented [2-4].

Although a kind of liquid antenna, seawater antenna is also a kind of special dielectric resonance antenna, it has some new characteristics different from traditional dielectric resonance antenna, such as that it's much easier for seawater or salt water to process into the shape needed than normal medium or metal, that it's convenient to adjust the geometrical parameters of structure and the electromagnetic characteristics of material, that it's easy to control working frequency and bandwidth, and that it has a very good reconfigurability[6-8]. In addition to this, the seawater can be drained when the seawater antenna is not in use, to reduce ship RCS, and enhance the imperceptibility. It is reported that the seawater antenna developed by the United States navy is mainly used for transmitting and receiving VHF (30 $300 \mathrm{MHZ})$ and UHF signals $(300 \sim 3000 \mathrm{MHZ})$. In this paper, the seawater antenna simulation model is presented, and simulation is carried out. the influence of the seawater antenna structure parameters on the radiation characteristics such as return loss, radiation efficiency is analyzed. And the structure and design parameters of seawater antennas that are suitable to work in VHF frequency band, UHF frequency band, and both VHF and UHF frequency bands are put forward.

\section{Simulation Model of Seawater Antenna}

An ideal cylindrical seawater antenna model is established in this paper, as shown in figure1. The bottom layer is a cuboid ( $300 * 300 \mathrm{~mm}, 1 \mathrm{~mm}$ thick) ground plate made of PEC (Perfect Electric Conductor), on which there is a cuboid $(100 * 100 \mathrm{~mm}, \mathrm{t} \mathrm{mm}$ thick) paxolin (phenolic laminate) substrate to separate seawater from ground plate. At the top, there is a PVC (Polyvinyl chloride) hollow tube $(300 \mathrm{~mm}$ in height, $\mathrm{d} \mathrm{mm}$ in inner diameter, and $2 \mathrm{~mm}$ in wall thickness). The antenna is fed with a PEC probe from the bottom. The inner diameter of the probe is $3 \mathrm{~mm}$, and the outer diameter is $9 \mathrm{~mm}$. The PVC tube filled with seawater acts as antenna. And the length of the part inside the seawater of the probe is $1 \mathrm{~mm}$. The height of seawater in the tube is $\mathrm{h} \mathrm{mm}$. 


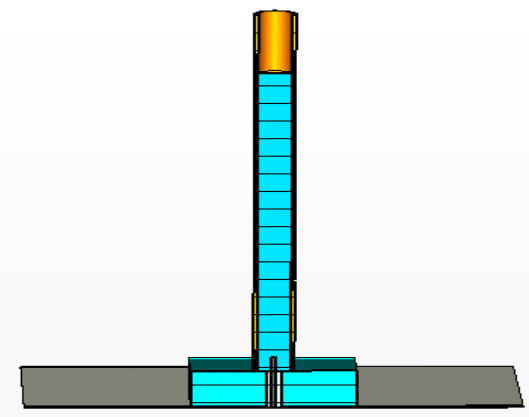

Fig.1 seawater antenna simulation model structure

\section{Analyzing of Seawater Antenna Radiation Characteristics}

In this paper, the optimization design and simulation are performed under the condition of a given initial values. The initial values are defined as shown in table 1 . The five factors which have a main influence on radiation characteristics of the seawater antenna are studied through parameter sweep, such as the seawater antenna length (or height) h, the substrate thickness t, the electrical conductivity $\sigma$ of seawater, the diameter $d$ of antenna, the length 1 of the feed probe, etc. The effects of each parameter on the performance are analyzed.

Table 1. initial values of antenna parameter

\begin{tabular}{|l|c|c|c|c|c|}
\hline \multirow{2}{*}{ initial values(mm) } & $h$ & $t$ & $\sigma$ & $d$ & $l$ \\
\cline { 2 - 6 } & 270 & 30 & 25 & 21 & 11.5 \\
\hline
\end{tabular}

\subsection{Effect of Height on Radiation Characteristics of Seawater Antenna}

Taking the values in Table 1 to do the simulation, and varying the parameter $\mathrm{h}$ from $250 \mathrm{~mm}$ to $290 \mathrm{~mm}$ to study the return loss S11 parameter (as shown in figure 2) and the radiation efficiency $\eta \mathrm{rad}$ (as shown in figure 3) of the seawater antenna. It is observed from the S11 curves that $\mathrm{h}$ determines the working resonant frequency of seawater antenna, and it also has an effect on S11 amplitude. And it is shown from the radiation efficiency curves that $h$ has an effect on $\eta \mathrm{rad}$ to a certain extent in the $0.4 \mathrm{G}$ to $1.6 \mathrm{G}$ frequency range, but there is no obvious regularity.

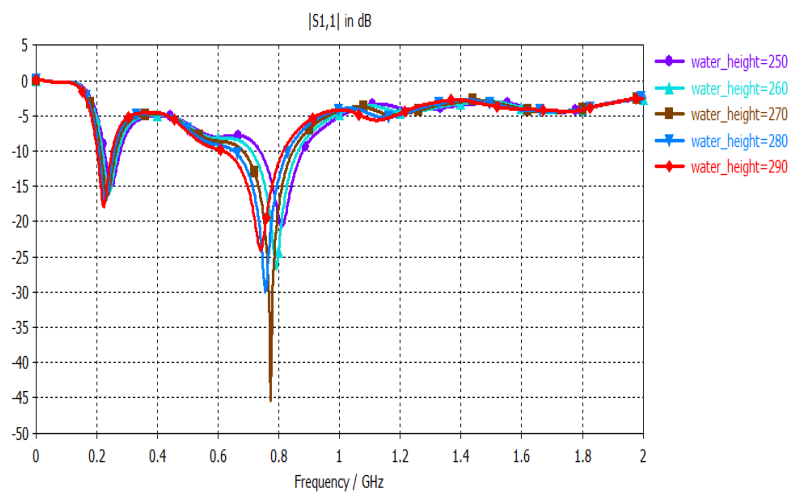

Fig.2 S11 parameter features on seawater antenna height change 


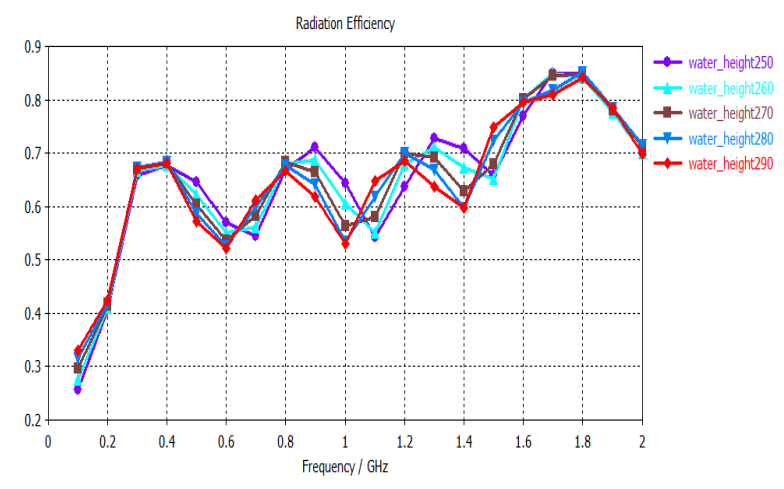

Fig. 3 radiation efficiency features on seawater antenna height change

\subsection{Effect of Substrate Thickness on Radiation Characteristics of Seawater Antenna}

Varying the substrate thickness $\mathrm{t}$ from $10 \mathrm{~mm}$ to $40 \mathrm{~mm}$ to study $\mathrm{S} 11$ parameter and radiation efficiency $\eta \mathrm{rad}$, as shown in figure 4 and figure 5 . The S11 curves in figure 4 show that $\mathrm{t}$ has a considerable influence on S11 curves. The change of $t$ causes a shift of the second resonant frequency, and also has a great effect on the distribution of S11 amplitude. It is observed from the radiation efficiency curves that $t$ affects $\eta \mathrm{rad}$ in high frequency range greatly. In a certain range, $\eta \mathrm{rad}$ has a regularity of increasing with the increase of $t$.

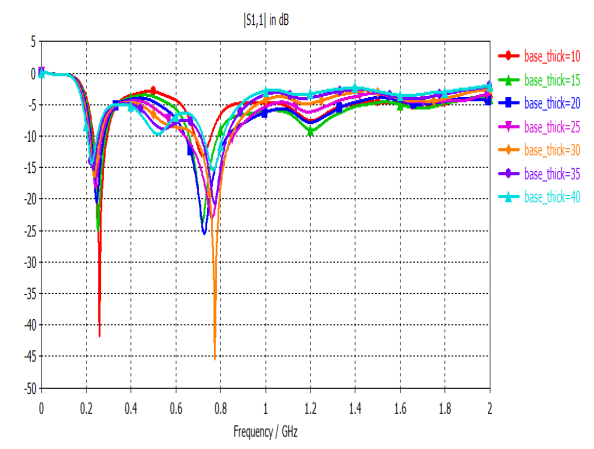

Fig.4 S11 features on substrate thickness change

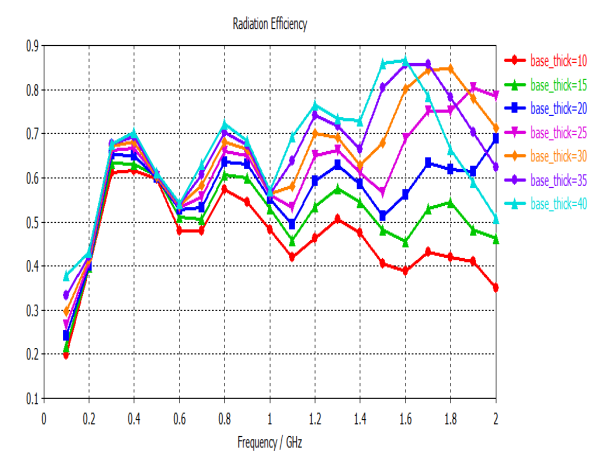

Fig.5 radiation efficiency features on substrate thickness change

\subsection{Effect of Seawater Electrical Conductivity on Radiation Characteristics of Seawater Antenna}

Varying the electrical conductivity $\sigma$ of seawater from 14.1 to $47 \mathrm{~s} / \mathrm{m}$ to study S11 parameter and radiation efficiency $\eta \mathrm{rad}$, as shown in figure 6 and figure 7. The S11 curves in figure 6 show that $\sigma$ mainly affects the amplitudes at the first resonant frequency points on the S11 curves. And the radiation efficiency curves show that $\sigma$ has a great effect on $\eta \mathrm{rad}$, and $\eta \mathrm{rad}$ increases with the increase of $\sigma$ in the whole frequency range. 


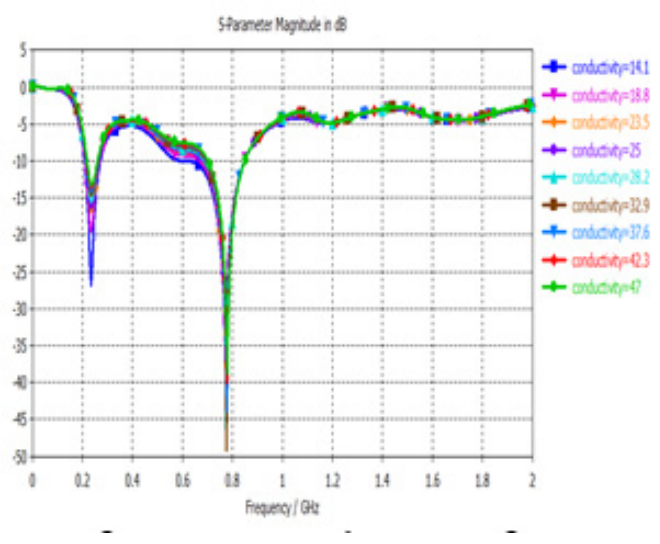

Fig. 6 S11 features on change of seawater electrical conductivit

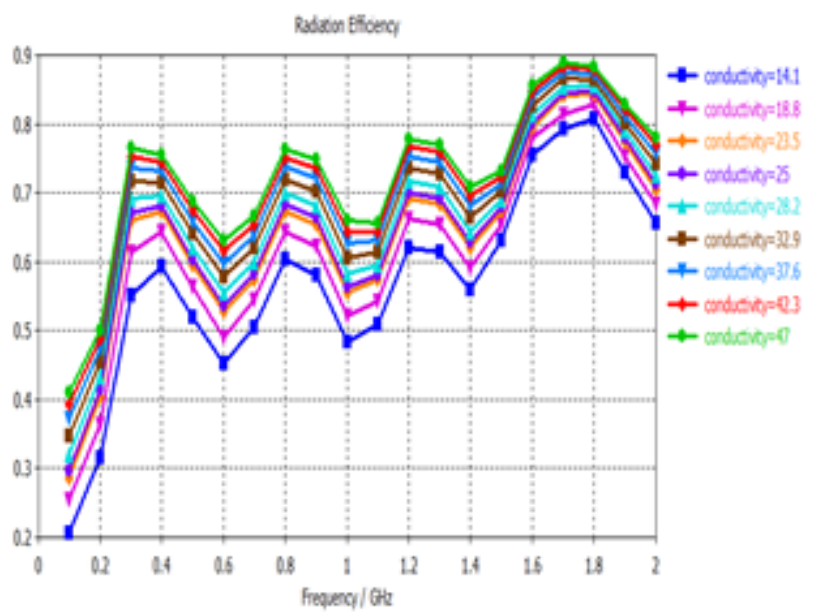

Fig.7 radiation efficiency features on change of seawater electrical conductivity

\subsection{Effect of Antenna Diameter on Radiation Characteristics of Seawater Antenna}

The results are shown in figure 8 and figure 9 . by varying the diameter of seawater antenna $\mathrm{d}$ from $18 \mathrm{~mm}$ to $24 \mathrm{~mm}$. The $\mathrm{S} 11$ curves in figure 8 show that $\mathrm{d}$ mainly affects the amplitudes at the both two resonant frequency points on the S11 curves. The radiation efficiency curves show that $\mathrm{d}$ affects $\eta \mathrm{rad}$ in low frequency range, and $\eta \mathrm{rad}$ slightly increases as $\mathrm{d}$ increases.

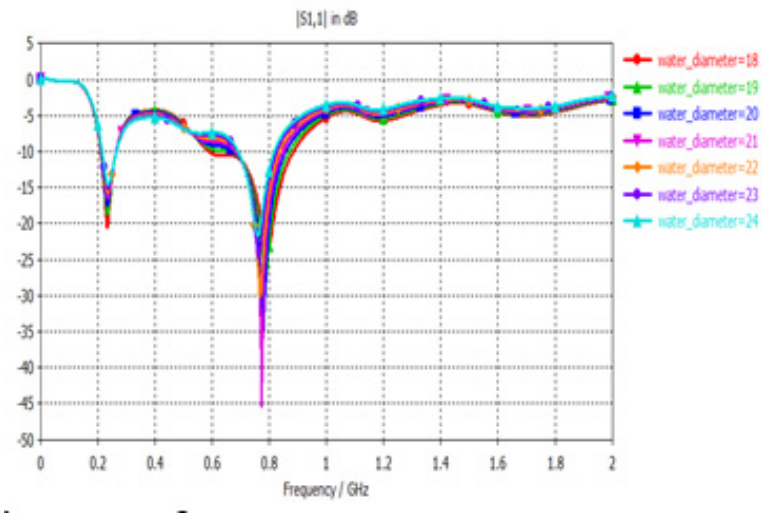

Fig.8 S11 features on seawater antenna diameter change 


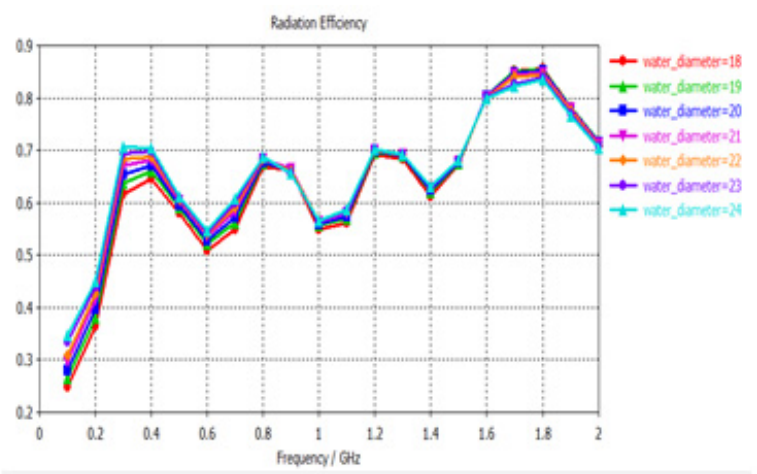

Fig.9 radiation efficiency features on seawater antenna diameter change

\subsection{Effect of the Length of the Feed Probe}

The curves in figure 10 shows the effect of the feed probe length 1 on S11 parameter, in fig.10 1 increases every $1 \mathrm{~cm}$ from $8.5 \mathrm{~cm}$ to $18.5 \mathrm{~cm}$. It is shown that the increase of 1 nearly has no impact on S11 curve features. the conclusion is in a good agreement with those that have been reported. Since the coincidence degree of S11 curves is very high, the analysis of effect of 1 on radiation efficiency of antenna is not performed in this paper.

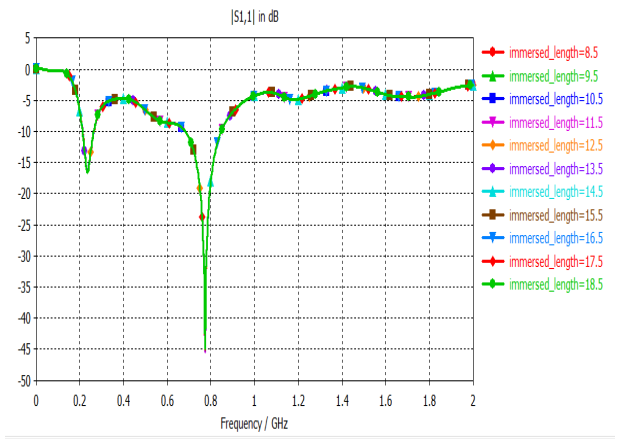

Fig.10 S11 features on feed probe length change

\section{Conclusion}

The height of seawater (h) mainly affects the position of resonant frequency. With the increase of $\mathrm{h}$, the resonant frequency decreases and the number of resonant frequencies increases as well. The substrate thickness ( $\mathrm{t}$ ) greatly affects the amplitude and the position of resonant frequency on S11 curve, but it's not a simple linear relationship. In low frequency range, the radiation efficiency increases with the increase of $t$. The seawater electrical conductivity $(\sigma)$ has a great impact on the radiation efficiency of the seawater antenna. As $\sigma$ increases, the radiation efficiency increases. The diameter of the seawater antenna (d) affects the amplitude of resonant frequency on S11 curve greatly, too. But it has little impact on the position of resonant frequency on S11 curve. The length of the feed probe (1) nearly has no impact on S11 parameter in a certain wide band.

According to the simulation analyses, the structure and design parameters of seawater antennas that are suitable to work in VHF frequency band, UHF frequency band, and both VHF and UHF frequency bands are presented in Table 2 .

Table 2. seawater antenna size in different frequency band

\begin{tabular}{|c|c|c|c|}
\hline Structure parameters & antenna in VHF & Antenna in VHF-UHF & Antenna in UHF \\
\hline Height $\mathrm{h} / \mathrm{mm}$ & 270 & 270 & 270 \\
\hline Substrate thickness t/mm & 10 & 20 & 30 \\
\hline seawater electrical conductivity $\sigma \mathrm{s} / \mathrm{m}$ & 25 & 25 & 25 \\
\hline Antenna diameter d/mm & 18 & 20 & 21 \\
\hline Length of feed probe $1 / \mathrm{mm}$ & 11.5 & 11.5 & 11.5 \\
\hline
\end{tabular}




\section{References}

[1]. Paraschakis E, Fayad H, Record P., Ionic liquid antenna, antenna technology: small antennas and novel metanmeaterials, IWAT, Italy, 2005.

[2]. Faya H, Record P., Broadband liquid antenna, Electronics Lett., 2006, Vol.12, No.1, pp.342.

[3]. Hazem Fayad and Paul Record, Wideband Dielectric Resonator Antenna with Reconfigurable Radiation Pattern, 2007.

[4]. Hua CZ; Shen ZX; Lu J; High-Efficiency Sea-Water Monopole Antenna for Maritime Wireless Communications, IEEE Trans. On Antennas and Propagation, 2014, Vol.62, NO.12, pp.59-68.

[5]. Junker, P.G., et al.: 'Effect of an air gap around the coaxial probe exciting a cylindrical dielectric resonator antenna', Electron. Lett., 1994, Vol30, (3), pp. 177-178.

[6]. Lei Xing, Yi Huang, Saqer S. Alja'afreh, Steve J. Boyes, A Monopole Water Antenna, Department of Electrical Engineering and Electronics University of Liverpool Liverpool, United Kingdom, November 2012. 